\title{
A NOVEL FRAMEWORK BASED ON THE IMPROVED JOB DEMANDS-RESOURCES (JD-R) MODEL TO UNDERSTAND THE IMPACT OF JOB CHARACTERISTICS ON JOB BURNOUT FROM THE VIEW OF EMOTION REGULATION THEORY
}

\author{
Naiding Yang ${ }^{1,2}$, Jintao Lu ${ }^{1}$ \& Jinfu Ye ${ }^{1}$ \\ ${ }^{1}$ School of Management, Northwestern Polytechnical University, Xi'an, China \\ ${ }^{2}$ Emergency Management Institute, Northwestern Polytechnical University, Xi 'an, China
}

received: 16.11 .2017 ;

revised: 5.2.2018;

accepted: 15.2.2018

\section{SUMMARY}

Background: It has been suggested that individual job characteristics have a significant impact on job burnout, and the process is subject to the regulation of demographic variables. However, the influence path of job characteristics on job burnout is still a "black box".

Subjects and methods: On the basis of a systematic literature review by employing Pub Med, Science Direct, Web of Science, Google Scholar, CNKI and Scopus for required information with the several keywords "Job burnout", "Emotion regulation", "Personality traits", and "Psychological stress", in this study, an improved mine rescue workers-oriented job demands-resources $(J D-R)$ model was put forward. Then, a novel analysis framework, to explore the impact of job characteristics on job burnout from the view of emotion regulation theory, was proposed combining the personality trait theory.

Results: This study argues that job burnout is influenced by job demands through expressive suppression and by job resources through cognitive reappraisal respectively. Further more, job demands and job resources have the opposite effects on job burnout through the "loss-path" caused by job pressure and the "gain-path" arised from job motivation, respectively. Extrovert personality traits can affect the way the individual processes the information of work environment and then how individual further adopts emotion regulation strategies, finally resulting in indirectly affecting the influence path of mine rescue workers' job characteristics on job burnout.

Conclusions: This present study can help managers to realize the importance of employees' psychological stress and job burnout problems. The obtained conclusions provide significant decision-making references for managers in intervening job burnout, managing emotional stress and mental health of employees.

Key words: Job burnout - Emotion regulation - Personality traits - job characteristics - Psychological stress

\section{INTRODUCTION}

With the continuous advancement of science and technology and the cumulatively social activities in recent years, human beings have been facing the increasingly frequent occurrence of all kinds of emergencies in the world while sharing social civilization brought by scientific \& technological achievements. For example, in the nuclear leakage disaster at the Fukushima nuclear power plant in Japan in 2011, 19 rescue workers were exposed to nuclear radiation, resulting in the emergency evacuation of hundreds of thousands of people from Fukushima. Subsequently, the nuclear proliferation crisis even aroused the nuclear radiation panic in neighboring countries. The massive gas explosion occurred in 2005 at Sunjiawan coal mine in Fuxin City of China killed Chinese coal miners and injured 30 others, and especially in recent years, since emergency accidents occurring in China's coal mines are frequently reported, coal mine accidents has become a hot topic for international discussion in the industrial and academic circles (Lu et al. 2014).
After the occurrence of coal mine disasters, mine rescue workers need to immediately engage in emergency response tasks in the shortest time. However, due to the unpredictability of the accident cause and the onsite work environment, mine rescue workers need to bear greater psychological pressure and emotional stimulation, thereby resulting in psychological stress reactions such as physical fatigue and cardiac exhaustion, including depression, anxiety, nervousness, sleep disorder and coronary disease, and psychological stress problems are subsequently widespread in mine rescue teams ( $\mathrm{Li}$ et al. 2012). However, the problem of job burnout caused by psychological stress is often overlooked by managers and mine rescue workers. Besides, the instability of psychological stress is just one of the reasons that affect work performance \& rescue effect, and cause safety accidents. Furthermore, several studies have shown that ( $\mathrm{Li}$ et al. 2012) most of the objects suffering job burnout caused by psychological stress are front-line rescue workers. At the same time, these rescue workers have the following common features in terms of work environment and work nature: boring and poor working environment, higher work intensity and 